\title{
Marie von Ebner-Eschenbach, Tagebuch einer modernen Europäerin
}

\author{
Eleonora Jeřábková
}

\begin{abstract}
Annotation
Im Text werden Ausschnitte aus den Tagebüchern von Marie von Ebner-Eschenbach zitiert. Weil es sich nur um eine kurze Demonstration handelt, haben wir das Jubiläumsjahr 1866 gewählt. Marie von Ebner-Eschenbach beschreibt die Ereignisse in der Weltpolitik, zu Hause, ihre eigene Tätigkeit und schafft so ein wahres Bild der damaligen gesellschaftlichen Situation.

The article contents parts of the writer's Marie von Ebner-Eschenbach diaries. The year 1866 was chosen from these diaries according to the jubilee. The author describes events in the world politics, at her home, describes her creative work and life of all dweller strata in the region of her native Zdislavice. In this way she creates the picture of the social life at those times.
\end{abstract}

Im Jahre 2016 erinnern wir uns des 100. Todestags der Schriftstellerin Marie von EbnerEschenbach. Sie wurde im Jahre 1830 in Zdislawitz in Mähren geboren und starb in der Mitte des Krieges im Jahre 1916. Die bei uns fast vergessene Autorin befasste sich in ihrem Werk sehr intensiv mit dem Leben der Menschen, die in Mähren, damals in der Österreichischen Monarchie, wohnten. Sie umfasste fast alle gesellschaftlichen Schichten ihrer Umgebung. Es waren Adelige, Intellektuelle aber auch gewöhnliche Menschen auf dem Dorfe. Ihre Schicksale entwickeln sich in ihren Büchern mit tiefer Psychologie, in feinen Details kritisch aber auch liebenswürdig. Der Leser kann durch ihre Worte auch die herrliche Natur der Gegend oder die Lebensart in einer Stadt, meistens Wien, genießen. Sie beschreibt die einzelnen Ereignisse oder den ganzen Lebenslauf einer Gestalt, die sich in einem gewissen gesellschaftlichen Milieu befindet, dem sie sich auch moralisch verpflichtet fühlt oder gegen dessen Gesetze sie sich zu wehren versucht. Ein feiner, psychologischer Realismus, obzwar in der Zeit einer starken Entwicklung vom Naturalismus. Ebner liest Zolas aber auch Dostojevskis Bücher. Wehrt sich aber dem Gedanken, dass ein menschliches Wesen sein Schicksal durch eigene Kraft nicht bewirken kann. Dieses Problem spielt z.B. die Hauptrolle in ihrer Novelle Das Gemeindekind ${ }^{1}$. Pavel, die

1 Das Gemeindekind, Erzählung, Berlin 1887 
Hauptgestalt, obwohl aus schlimmen familiären Verhältnissen stammend und in schwieriger Lage lebend, entwickelt sich in einen aufrichtigen und selbstbewussten Menschen. Sogar auch gereifter Mensch kann seine Meinungen und seine Lebensweise ändern, wie wir es bei Doktor Rosenzweig in der Novelle Der Kreisphysikus ${ }^{2}$ beobachten können. Ihre Gestalten stehen auch nicht mit Gleichgültigkeit gegenüber den Meinungen ihrer Umgebung. Das zeigt die Autorin vor allem in ihren dialogisierten Novellen, wie zum Beispiel Die Bettelbriefe ${ }^{3}$, oder in dem Einakter Am Ende $e^{4}$. Diese tiefen Menschkenntnisse sind vielleicht das Kostbarste, was das Werk der Schriftstellerin für ewig geltend macht. Es steht im Hintergrund ihrer Prosa, ihrer Theaterstücke aber auch in ihren Parabeln und vor allem in den Aphorismen, die nicht nur belehrend, sondern auch ermutigend auf den Leser wirken.

Wie war also das Leben von Marie von Ebner-Eschenbach, wie war die Autorin dieser Texte in ihrem Privatleben. Das waren die Fragen, die wir uns gestellt haben, als wir eine Ausstellung über sie vorbereitet haben. Man kann einen Autor durch sein Werk präsentieren, man kann aber weiter darüber nachdenken, wer dieser Mensch war, der es geschrieben hat. Und da bot sich die Gelegenheit, eben einen Schritt in das Private zu machen. In dem Mährischen Landesarchiv in Brno werden einige von den Tagebüchern der Autorin aufbewahrt. Eine seltene Kostbarkeit aus mehreren Hinsichten. Wir wissen also, dass die Autorin in Zdislawitz als Gräfin Marie Dubsky geboren wurde. Sie war die Tochter von Marie von Vockel. Diese Familie kam nach Mähren aus Sachsen und begann in Mähren mit der Schafzucht. Großvater Vockel baute das Gebäude in Zdislawitz in ein schönes Barockschloss um und erhob das Niveau der Umgebung von Zdislawitz in ein gut bewirtschaftetes Land. Ihr Vater war Franz Graf Dubsky, aus einem alten tschechischen protestantischen Zweig der Familie Dubsky aus Trebomyslitz stammend, der sein durch die Heirat gewonnenes Gut weiter im guten Sinne führte. Bald nach der Geburt von Marie starb ihre Mutter. Das Mädchen wurde von mährischen tschechisch sprechenden Ammen erzogen und auch die folgenden Stiefmütter bewirkten positiv ihre Entfaltung. Etwas war aber anders als bei den anderen Geschwistern. Es war die Überzeugung, dass man im Leben etwas tun soll, womit man die Anderen beeinflussen kann. Am Anfang war es das Theater, in dem sich eigentlich alle Künste verbinden. Musik, Bewegung, Bild und vor allem das Wort. Die besten Theaterstücke zu schreiben, das war der Wunsch der jungen Literatin. Gewiss haben es die regelmäßigen Theaterbesuche in Wien bewirkt, gewiss spielte hier eine wichtige Rolle die große Familienbibliothek, die zu Hause zur Verfügung stand. Die zweite Stiefmutter Xaverine Dubsky, geborene Kolowrat-Krakows$\mathrm{ky}^{5}$ schenkte ihrer Stieftochter nicht nur das gesammelte Werk von Friedrich Schiller, sie gab sogar die Texte von Marie dem damals hochgeschätzten Franz Grillparzer ${ }^{6}$ zu lesen. Allerdings der erste und für viele Jahre auch letzte literarische Erfolg von Marie. Bald

2 Der Kreisphysikus, Erzählung, Berlin 1883

3 Die Bettelbriefe, dialogisierte Novelle, Wien 1898

4 Am Ende, Szene in einem Aufzug, Berlin 1897

5 Xaverine Dubsky, geborene Kolowrat-Krakowsky, 1808-1869

$6 \quad$ Franz Grillparzer, 1791-1872, Dramatiker, Dichter, Literaturkritiker 
darauf sollte sie nämlich an schlechte Kritiken und vor allem ernste Proteste der Familie gegenüber ihrem Schreiben stoßen. Vor allem ihr Mann Moriz von Ebner-Eschenbach ${ }^{7}$, den sie im Jahre 1848 heiratete und der eigentlich bis zu seiner Pensionierung im Jahre 1874 seine Karriere an der Militärakademie aufbaute, versuchte mit allen Mitteln seine Frau von ihrem Schaffen abzuhalten. Wir wissen, dass die Schriftstellerin alle diese Hindernisse überwunden hat und wirklich noch während ihres Lebens zu einer von den anerkanntesten Literaten in der deutschen Sprache wurde. Sie gab ihre Werke aus, fand viele Leser, als erste Frau bekam sie an der Wiener Universität den Ehrendoktor und im Jahre 1911 wurde sie sogar für den Nobelpreis nominiert. Das alles kostete sie aber viel Geduld, eine gewisse Hartnäckigkeit, Bildung, Demut und Entschlossenheit. Und das sind eben die Punkte, die wir in den Tagebüchern finden können. Dabei aber verliert sie nicht das Interesse an ihren Mitmenschen. Sie wird zu einer guten Ehefrau, einer hervorragenden Tante aller ihrer Nichten und Neffen, zu einer hilfsbereiten Freundin von vielen Menschen, die sich an sie mit Vertrauen gewendet haben. Versuchen wir also ein bisschen in den Tagebüchern zu blättern. Wie schon gesagt wurde, befindet sich ein Teil der Hefte in dem Mährischen Landesarchiv in Brno. Die ersten Anmerkungen beginnen im Jahre 1863, es sind meistens nur kurze Sätze vor allem auf ihre literarische Tätigkeit konzentriert. Im Jahre 1864 werden es schon längere Abschnitte, die auch andere Informationen beinhalten. Langsam findet die Autorin ihre eigene Art, um die Tage und Ereignisse zu beschreiben. Sie widmet sich der Beschreibung der Naturverhältnisse, ihrer Gesundheit und den gesundheitlichen Zuständen ihrer Verwanden oder Freunde. Manchmal zitiert sie kurze Abschnitte aus Gesprächen, die sie für wichtig hält. Sie widmet sich auch dem eigenen Schaffen und beschreibt das Werk, an dem sie arbeitet, oder die darauf folgenden Reaktionen. Immer mehr reagiert sie auch auf gesellschaftliches Geschehen in der Monarchie aber auch in anderen Ländern Europas, später auch in Amerika. Oft und gerne schreibt sie meistens kritische Bemerkungen zu verschiedenen Theatervorstellungen in Wien, die sie besucht hat. Sie äußert auch Meinungen über Bücher und ihre Autoren, die sie gerade liest. Täglich notiert sie auch von wem sie Post bekommen hat und an wen sie geschrieben hat. So entstehen Aufzeichnungen, die nicht nur für die Literaturwissenschaftler, sondern auch Historiker oder Psychologen interessant sind. Manche Stellen sind ausradiert oder korrigiert, entweder von der Hand der Autorin selbst oder von jemandem aus der Familie. Die Schriftstellerin dachte bestimmt darüber nach, ihre eigenen Erinnerungen in Buchform herauszugeben, ein Teil von dieser Idee wurde dann auch später in dem Buch Meine Kinderjahre ${ }^{8}$ realisiert. Längere Passagen sind meistens sehr leserlich geschrieben und man kann sich gut vorstellen, dass die Tagebücher auch als Buch erscheinen könnten. Für die deutschsprachige Welt wurden sie schon von Karl Konrad Pohlheim ${ }^{9}$ mit Kommentaren herausgegeben. Sie beinhalten aber auch die Tagebücher, die bis zum vorletzten Tag des Lebens von Marie von Ebner-Eschenbach geschrieben wurden. In den Jahresbüchern, die sie sich zu

7 Moriz von Ebner-Eschenbach, Ehemann und Cousin von Marie von Ebner-Eschenbach, 1815-1898

8 Meine Kinderjahre, Biographische Skizzen, Berlin 1906

9 Marie von Ebner-Eschenbach, Kritisch herausgegeben und kommentiert von Karl Konrad Pohlheim, 1989, 1991, 1993, 1995, 1997 Tübingen, Niemeyer 
diesem Zwecke gekauft hat und die eigentlich als ein Kalender dienen sollten, finden wir nicht nur Tagesaufzeichnungen, sondern am Ende auch Rechnungen, Zitate von anderen Schriftstellern, Philosophen oder auch Politikern. Manchmal sind hier oder an der Stelle der gewissen Tage Zeitungsabschnitte eingeklebt, die sie für besonders wichtig hielt. Manchmal zeichnet sie Porträts, kleine Stillleben und Landschaften.

Um eine Vorstellung zu bieten wählten wir das Jahr 1866, denn es sind 150 Jahre nach der Schlacht bei Königgrätz verflossen und die Aufzeichnungen von Marie von EbnerEschenbach sind in diesem Sinne sehr bereichernd. Die sechsunddreißigjährige Dramatikerin ist völlig auf ihre Arbeit konzentriert, stößt aber auf den Unwillen ihres Mannes.

\section{Jänner}

Spät aufgestanden. Abends im Theater... Nach dem Theater hatten wir eine lange Beratung über meine unglückliche Schriftstellerei. Warum muss ich gerade Theaterstücke schreiben wollen? Lieber gescheite Romane.

Wenn ich Moriz versprechen könnte, dass ich keine Theaterstücke mehr schreiben will, alles wäre gut. Aber kann ich?

An Sophie Erdödy. Ihre Schwiegermutter gestorben. An Marie. Von Marie.

Schon bald darauf aber erfahren wir, dass diese Beratung für Moriz nicht erfolgreich war:

\section{Februar}

Das gekürzte Manuskript „Versuchung“ nach Prag an Herrn John geschickt. Im Laufe des Vormittags Poli, Leo, Sefine, Victor ${ }^{10}$, der heut abends den Cottillon mit L. tanzt und hofft Gelegenheit zu finden, ihr die Absicht seines Hierherkommens mitzuteilen. Großmama, die ich besuchte, wäre entzückt; wen dieser Heirat sich machen würde, ich glaube nicht, dass es der Fall sein wird...

\section{Februar}

...Die Antwort Resis lautet „L. ist nicht frei!“ doch verbarg sie mir nicht, dass die Heirat, die bevorsteht, nicht aus Neigung geschlossen wird. Wenn ich Victor wäre, und wollte!

Noch lebt man im Frieden, keine Gedanken an den Krieg, man plant schon die große Ausstellung, die in Paris im Jahre 1867 stattfinden soll.

\section{März}

Vormittag nicht besonders fleißig gewesen, weil ich starke Kopfschmerzen hatte. Moriz mit Comisi abgeholt u. mir die Gegenstände angesehen, die er zur Pariser Ausstellung ${ }^{11}$ schicken will. Bei den Eltern gespeist. Abends bei Kress.

An Marie, Adolf ${ }^{12}$ und Victor (zum Geburtstag). Von Julie Erdödy- in der Haarwuchsangelegenheit. Sie schickt mir Mamas Briefe an sie. Schrecklich! Schrecklich dumm!

10 Victor Dubsky, Halbbruder von Marie von Ebner-Eschenbach, 1834-1915

11 Zweite Pariser Weltaustellung, 1. 4. - 3. 11. 1867 Champ-de-Mars, Exposition universelle d'Art et d'industire

12 Adolf Dubsky, Halbbruder von Marie von Ebner-Eschenbach, 1833-1911 


\section{März}

Benedek in Wien.

Nachmittags bei der guten Friederl ${ }^{13}$, Geburtstag der beiden Brüder. Gott segne sie, erhalte sie und mir ihre Liebe.

In der Früh in die Stadt gegangen, bei Großmama Amelie Hartig abgeholt und mit ihr zu den Eltern. Dann zu Frau Litrow.

Nachmittags bei Sefine und Poli Stahl, die ich allein mit dem armen Claudius fand. Er stickte an einem furchtbaren Napoleons Kopf... Welche Geschmackverwirrung! Das kann man schon ein krankhaftes Gelüste nennen...

Die Familie Dubsky besuchte regelmäßig verschiedene Theatervorstellungen, es gehörte zum gesellschaftlichen Leben, aber auch zur Bildung vor allem der jungen Mitglieder der Familie. In der Schlossbibliothek in Lissitz befinden sich mehrere Manuskripte von Theaterstücken, die von den Dubskys für ihr Schlosstheater geschrieben wurden. Man lernte so Vieles, dachte aber nicht darüber nach, es wirklich ernst zu nehmen, wie es bei Marie Ebner der Fall war.

\section{März}

Ein guter fleißiger Tag.

Früh eine kleine Promenade, dann nach Hause und gearbeitet. Herrlich - ohne Unterbrechung. Bis zu Speisen bei den Eltern... Mama hatte die Loge im Burgtheater verkauft und eine auf der Wieden dafür genommen. Neue Versicherungen, dass es sie krank mache, wenn ich dasitze, wie ein alter boshafter Rezensent und mich im Kritisieren der Stücke und Schauspieler ergehe...

Interessant ist, dass gerade im Jahre 1866 das historische Drama Marie Roland ${ }^{14}$ entsteht. Die Gedanken über die Französische Revolution und das Verschwinden von Idealen wird hier ganz deutlich dargestellt. Das Gespräch zwischen Marie Roland und Danton zeigt deutlich, wie der Machtwille über den humanen Idealen siegt. Der Vergleich des Schicksals von Marie Roland und der Königin Marie Antoinette war für das damalige österreichische Publikum noch unakzeptabel.

\section{März}

... Vormittag Frau von Littrow. Ich habe ihr den ersten Aufzug der Roland vorgelesen. Wenn alle Leute, die das Stück sehen, so davon hingerissen werden, dann wackelt Schillers Thron im Himmel. Schade, dass man von ihr kein richtiges Urteil haben kann.

Langsam aber spürt man, dass sich etwas in Europa rührt, was viele Schicksale ändern wird.

10. April

Preußen alliiert sich mit Italien, das sich zum Kriege gegen uns verpflichtet, wenn er innerhalb der nächsten 3 Monate ausbricht. Dafür bekommen die Italiener Venedig von den treuen Preußen geschenkt. Zähneknirschend hört man das.

13 Friederike Kinsky (geborene Dubsky), Schwester von Marie von Ebner-Eschenbach, 1825-1895

14 Marie Roland, Trauerspiel in 5 Aufzügen, 1867 
Nichts aber hindert die Künstlerin daran, mit ihrer Arbeit aufzuhören und das Interesse an der Wissenschaft zu verlieren.

\section{April}

Den dritten Aufzug der M. Roland beendet. Abends Zimmermann. Litrows, Weils. Sehr spät Julie, die morgen abreist.

Bevor die Andern kamen, sprach Zimmermann sehr interessant über Schopenhauer und Fichte. Der Pessimismus des ersten, der subjektive Idealismus des zweiten. Wir sind von der Existenz keines außer uns liegenden gewiss - die Außenwelt nur eine Vorstellung.

Die kriegerischen Ereignisse tauchen aber immer mehr auf.

\section{April}

...Zu den Eltern zum Speisen. Garibaldi beginnt sich in Italien zu regen, die Verbündeten unseres Brudervolks schlagen noch vor ihm los...

\section{April}

Spät aufgestanden. Wieder recht leidend. Vormittags nicht recht fleißig. Fühle mich so unwohl, dass mich nicht einmal die Arbeit zerstreut. Schlechtes Wetter, schneit und regnet. Moriz ungeheuer beschäftigt mit der Ausführung seiner Torpedos. Der Gedanke an die Reise nach England ist aufgegeben und es gilt jetzt mit größter Beschleunigung die Küsten und Häfen Dalmatiens, Venedig und Triest mit Seeminen zu verteidigen.

\section{Mai}

...Bei Hof wissen sie ebenso wenig wie wir armer Plebs.

Und der Krieg betrifft allmählich die ganze Familie.

\section{Mai}

Die Mutter schreibt sehr ruhig, sehr gefasst. Sie fuhr nachdem sie durch Heinrich ${ }^{15}$ seinen bevorstehenden Abmarsch erfahren, nach seiner Station. Er war aber bereits seit 3 Stunden fort. Auch Friederl schreibt mutig und entschlossen. Sie bliebe trotz des Krieges gerne in Bürgstein.

Um 3 Uhr Nachmittag kam Ida Fleischl ${ }^{16}$ und ich las ihr in einem Atem die Roland vor. Es muss eine tüchtige, starke, tieffühlende Natur sein, die ein fremdes Werk mit solchem Antheil mit so energischem Interesse in sich aufnehmen und überblicken kann.

\section{Juni}

...Die Zeitungen sprechen von nichts als von der Konferenz. Nächste Woche soll sie zusammentreten. Bis 15. Juni greift keine der kriegsbereiten Mächte an. Die Geschicke Europas sind in Napoleons Händen...

15 Heinrich Dubsky, 1847-1927, Halbbruder

16 Ida Fleischl-Marxow, 1825-1899, Freundin von Marie von Ebner-Eschenbach und Mäzenatin von der Dichterin Betty Paoli. 
16. Juni

Fürchterliche Kopfschmerzen. Adolf und Victor beim Speisen. Adolf reist heute wieder nach Hause. Beide Brüder sehr unglücklich darüber, dass sie noch keine Aussicht haben ins Feld geschickt zu werden. Nicht ein Torpedo gelegt, das Kabel nicht angekommen!

\section{Juni}

... Victor und Moriz in der Stimmung, die man sich denken kann - es ist auch entsetzlich. Heute hat unser Kaiser das Kriegsmanifest erlassen.

19. Juni

Befehl zum Vormarsch der Armee nach Böhmen.

Abschied von Victor genommen, er geht heut um ein Uhr Nachmittag nach München.

Von der Mutter. Heinrich war in Zdislawitz.

23. Juni

Bucsan.

...Keine Neuigkeiten in der Zeitung. Beim Speisen Zichys aus Pistjan. Blieben bis 7 Uhr. Ich recht dumm und langweilig, der Kreis der Menschen, mit denen ich umgehen kann, wird immer kleiner. Ist das recht! Gewiss nein! Ein großes Herz schließt sich nicht so karg von allen ab denen es nichts zu geben hat, auch denen die nichts von uns wollen, sollen wir unser warmes Interesse bewahren.

An Moriz, Friederl, Sofie.

Von Marie.

\section{Juni}

Früh Kirche. Abends mit Layos ausgeritten.

Marie die Roland vorgelesen. Einzelheiten gefielen ihr, für das Beste an dem Stück aber hat sie kein Auge.

25. Juni

Nachmittags die telegraphische Nachricht von dem Siege von Custozze. 2000 Gefangene...

Hoch Erzherzog Albrecht hoch unsere tapfere Armee. Gutes Vorzeichen für die Armee in Böhmen? Gott gebe! Gott gebe!

28. Juni

Angefangen zusammen zu lesen Auerbachs Auf der Höhe. Siegesnachrichten aus dem Norden. Bei Münchgrätz schlugen wir die Preußen. 23 Kanonen sind erobert. Nach Brestovan um dort die gute Botschaft zu verkündigen.

Kein Brief von Moriz.

29. Juni

...Abend sehr beunruhigende Nachrichten aus Böhmen.

Kein Brief von Moriz. 


\section{Juli}

Bei furchtbarer Hitze in die Kirche. Dann gelesen, Zeitungen und Briefe brachten heut nichts besonders und freundliches.

Tratenau! Skalitz!

Zum Verzweifeln.

Keine Nachrichten von Moriz.

\section{Juli}

Für mich ein Rasttag - für wie viele arme Menschen aber ein qualvoller Plagetag? Unsere Armee kämpft unablässig im Norden, es soll nicht gut gehen, Clam mit seinen Corps bei Münchengrätz zu spät gekommen sein. Erzherzog Leopold nach dem Gefechte in Skalitz zurück geschickt nach Pardubitz...

\section{Juli}

Layos kommt aus Pressburg zurück und bringt die Nachricht Heinrich Gudenus sei schwer durch 2 Lanzenstiche verwundet - Gott gebe dass uns bald tröstlichere Stunde werde. Es wäre zu schrecklich für die arme Großmama, in ihrem hohen Alter noch einen Enkel zu verlieren. Und Louise und Gabriel! - Welch ein garstiger Unsinn ist doch der Krieg!

\section{Juli}

Die unglückliche Nachricht der Niederlage unserer Armee in Böhmen. Alphons Dubsky ${ }^{17}$ tot. Benedeks ${ }^{18}$ Bericht an den Kaiser trostlos. Schnöde! Nach 5 stündigen glorreichen Gefechte fielen uns frische preußische Truppen in die Flanke und die Armee trat den Rückzug. Anfangs langsam, dann in rasender Eile an. Also schmachvollste Flucht.

Layos bricht in Tränen aus.

Es folgen weitere Berichte und Beschreibungen der Umstände am Kriegsfeld. Das bedeutet aber noch lange nicht das Ende des Unheils, denn während des Krieges bricht im Land die Cholera aus.

\section{September}

... Die Cholera reitet nicht. Doch spuckt sie in unserer Gegend.

In Hostitz heut 4 Leichen. Darunter die 16 jährige Tochter des Inspektors. Zum Speisen Melanie und Toni Bukuffsky, recht oberflächliche und unbedeutende Geschöpfe. Die Schönheit ohne wertvollen Hintergrund ist doch keine so allerweltbesiegende Gabe, als man in der Jugend wohl glaubt.

Von Moriz. Er kommt vielleicht schon in 8 Tagen zurück.

\section{September}

Heut vor 36 Jahren habe ich eine große Dummheit begangen, die nämlich: geboren zu werden. Die Strafe ist aber auch auf dem Fuß gefolgt.

17 Alfons Dubsky, Cousin aus dem Lissitzer Zweig der Familie Dubsky, 1843-1866

18 Ludwig Benedek, 1804-1881, österreichischer Feldherr 
Früh in die Gruft, es ist doch dort unten so übel nicht, wenn man nur die Tür offen stehen lässt und hinaus sehen kann in das frische Grün und den warmen Sonnenschein.

Nachmittag: erzählte der Jäger Trauriges von dem Herrschen der Cholera in Honietitz, in Hostitz ist die Frau des Inspektors Baronin Türkheim erkrankt...

\section{September}

...In Honietitz starben 5 Personen. Zdislawitz ist aber noch so ziemlich frei von der Cholera Von Moriz aus Venedig, kommt am 24.

\section{Oktober}

Sterbetag meiner Mutter.

Ich ließ eine Messe in der Kapelle lesen. Der Pfarrer erzählte während des Frühstücks viel Trauriges und Erschütterndes von den Verheerungen, welche die Cholera in seinem Pfarrsprengel angerichtet. Wir ahnten nicht, dass rings umher so viel Elend geherrscht - wir wollen davon nichts wissen und entheben uns somit der Verpflichtung zu helfen und zu trösten. Und wir wären beleidigt, wenn Einer uns sagte, dass wir keine Christen sind.

Die Schriftstellerin starb im hohen Alter, erlebte noch Vieles und immer tiefer und genauer beobachtete sie ihre Mitmenschen ohne einen Wert darauf zu legen, welcher Abstammung sie sind oder welche Position sie in der Gesellschaft vertreten. Auch ihre Tagebücher sind ein literarisches Werk, das direkt aus der Seele der Autorin kommt. Die Schrift deutet auf eine ausgeglichene Persönlichkeit und ihre Gedanken oder Bemerkungen tragen in sich etwas immer Aktuelles. Im April des Jahres 2016 wurden die Tagebücher zum archivalischen Kulturdenkmal der Tschechischen Republik ernannt. Nun wäre es auch gewiss zu empfehlen, wenigstens eine Auswahl aus diesen Texten ins Tschechische zu übersetzten.

\section{Literatur}

MARIE VON EBNER-ESCHENBACH: Tagebücher 1866, Handschrift, Archiv zdislavické větve rodiny Dubských, G 366, inv. číslo 543-547

PhDr. Eleonora Jeřábková, Ph.D. / jerabek.mojmir@volny.cz

Muzeum města Brna, Dům Jiřího Gruši

Hudcova 76, 60000 Brno, CZ 
